๑ Open Access Full Text Article

REVIEW

\title{
Current Challenges in the Recognition and Management of Delirium Superimposed on Dementia
}

\author{
Anita Nitchingham (D) ${ }^{1,2}$ \\ Gideon A Caplan (D) ${ }^{1,2}$ \\ 'The Prince of Wales Clinical School, \\ University of New South Wales, Sydney, \\ NSW, Australia; ${ }^{2}$ Department of Aged \\ Care, Prince of Wales Hospital, Sydney, \\ NSW, Australia
}

\begin{abstract}
Delirium occurring in a patient with preexisting dementia is referred to as delirium superimposed on dementia (DSD). DSD commonly occurs in older hospitalized patients and is associated with worse outcomes, including higher rates of mortality and institutionalization, compared to inpatients with delirium or dementia alone. This narrative review summarizes the screening, diagnosis, management, and pathophysiology of DSD and concludes by highlighting opportunities for future research. Studies were identified via Medline and PsycINFO keyword search, and handsearching reference lists. Conceptually, DSD could be considered an "acute exacerbation" of dementia precipitated by a noxious insult akin to an acute exacerbation of heart failure or acute on chronic renal failure. However, unlike other organ systems, there are no established biomarkers for delirium, so DSD is diagnosed and monitored clinically. Because cognitive dysfunction is common to both delirium and dementia, the diagnosis of DSD can be challenging. Inattention, altered levels of arousal, and motor dysfunction may help distinguish DSD from dementia alone. An informant history suggestive of an acute change in cognition or alertness should be investigated and managed as delirium until proven otherwise. The key management principles include prevention, identifying and treating the underlying precipitant(s), implementing multicomponent interventions to create an ideal environment for brain recovery, preventing complications, managing distress, and monitoring for resolution. Informing and involving family members or caregivers throughout the patient journey are essential because there is significant prognostic uncertainty, including the risk of persistent cognitive and functional decline following DSD and relapse. Furthermore, informal carers can provide significant assistance in management. Emerging evidence demonstrates that increased exposure to delirium is associated with neuronal injury and worse cognitive outcomes although the mechanisms through which this occurs remain unclear. Given the clinical overlap between delirium and dementia, studying shared pathophysiological pathways may uncover diagnostic tests and is an essential step in therapeutic innovation.
\end{abstract}

Keywords: delirium, dementia, delirium superimposed on dementia, ageing

\section{Introduction}

Delirium and dementia are commonly encountered by clinicians in both hospital and outpatient settings, and prevalence is rising amid global population ageing. Dementia, also known as major neurocognitive disorder, is chronic and characterized by decline in one or more cognitive domains, leading to functional dependence and a need for assistance with activities of daily living. ${ }^{1}$ Delirium is an acute and
Correspondence: Anita Nitchingham Department of Aged Care, Ground Floor Edmund Blackett Building, Prince of Wales Hospital, Barker Street, Randwick NSW, 203I, Australia

Tel +61293824252

Fax +6I29382424I

Email a.nitchingham@unsw.edu.au 
fluctuating disturbance in cognition, which predominantly affects attention and awareness and is precipitated by a medical illness or toxin. ${ }^{1}$

Despite their distinct diagnostic criteria, these conditions are epidemiologically and clinically interconnected. Specifically, each condition is the major risk factor for the other. Patients with delirium have 12 times greater odds of developing dementia than patients without delirium; this relationship is dose-dependent, with greater delirium exposure (recurrent episodes, increased severity, and longer duration) increasing the risk of future dementia., ${ }^{2,3}$ Similarly, patients with dementia are four times more likely to develop delirium, with increased severity of dementia conferring greater risk. ${ }^{4,5}$

Both conditions are characterized by cognitive dysfunction, albeit the onset of delirium is acute, resulting from noxious insults, while dementia is usually insidious and attributable to neurodegenerative genetic and lifestyle factors. The cognitive profiles of delirium and dementia may differ, particularly in the early stages of dementia; however, as dementia progresses, many patients experience neuropsychiatric symptoms, including hallucinations, delusions, aggression, and sleep disturbances, which are referred to as behavioral and psychological symptoms of dementia (BPSD). Since BPSD mimics delirium, differentiating delirium from dementia can be challenging even for discerning clinicians and in practice sometimes the only distinguishing feature may be the identification of a noxious insult triggering the change in cognition and function.

Although delirium is traditionally considered a reversible condition, for many patients, particularly those with underlying dementia, delirium may be irreversible, partially reversible, or persist for months. ${ }^{6,7}$ In this scenario, the two conditions merge, and long-lasting delirium becomes dementia.

Yet distinguishing delirium and dementia is critical. Delirium is a medical emergency and may be the only sign of acute illness; thus, failure to recognize and investigate delirium may have life-threatening consequences. Even so, $43-76 \%$ of delirium is unrecognized. ${ }^{8,9}$

Consequently, when delirium and dementia coexist, which is traditionally referred to as delirium superimposed on dementia (DSD), clinicians often face diagnostic and prognostic dilemmas. In patients with dementia, a family member or caregiver reporting an acute change in cognition or function should prompt further assessment for DSD.

Conceptually, DSD could be considered an acute exacerbation of dementia akin to acute exacerbations of cardiac or chronic obstructive pulmonary disease. The goal of therapy is to remove the external stressor causing decompensated organ function, manage the symptoms of the acute exacerbation, and monitor for resolution. ${ }^{10}$

Compared to dementia, research on delirium and particularly DSD is lagging but growing steadily. This review will focus on the epidemiological, pathophysiological, and clinical aspects of DSD. We conclude by highlighting gaps and opportunities for advancing knowledge in this field.

\section{Methods}

An initial Medline and PsycINFO search using the keywords of "delirium superimposed on dementia" (updated prior to submission, 1985- Mar 2021) yielded 184 articles that were screened by a single author (AN) for inclusion. Peerreviewed original research articles and systematic reviews focusing on the epidemiology, pathophysiology, management and outcomes of delirium superimposed on dementia were included. Literature reviews were also read and the references lists handsearched for relevant studies. A forward citation search was conducted on one systematic review related to screening tools ${ }^{11}$ to identify new studies in this area. Management principles from best practice guidelines were included. ${ }^{12,13}$ Articles written in languages other than English were excluded.

\section{Epidemiology of Delirium Superimposed on Dementia}

Although the prevalence of delirium in hospitalized patients varies depending on the population, in general, it affects up to one in four adult medical patients. ${ }^{14}$ The prevalence of dementia is also high, affecting 12.9-63\% general hospital patients $>55$ years old. ${ }^{15}$ Rates of DSD in older hospitalized patients range from $22 \%$ to $31 \%{ }^{16-18}$

Delirium and dementia are associated with lengthier hospital stays, functional decline, and increased mortality compared to cognitively intact inpatients. ${ }^{15,19-23}$ The outcomes for patients with DSD are even worse, and the risk of mortality at 12 months is double that of patients with delirium only and dementia but without delirium. ${ }^{24-27}$ Baseline dementia predicts delirium persistence, and DSD is associated with accelerated cognitive deterioration that continues for more than five years after adjusting for recurrent hospitalization, baseline cognitive function, and baseline rate of cognitive decline. ${ }^{7,28}$ Given the short- and long-term implications of DSD (longer hospital stay, use of community services, and increased need for residential aged care), the economic burden is substantial. ${ }^{29,30}$ 
Beyond this, delirium is a distressing condition for patients and their family members. ${ }^{31}$ Many patients with dementia recall aspects of their delirium episode, including feelings of confusion, fear, and associated distress. ${ }^{32}$ Caregivers also report moderate distress, particularly related to patients with prolonged inattention, disorientation, psychomotor retardation, and delusions. ${ }^{33}$

\section{Pathophysiology of Delirium Superimposed on Dementia}

Delirium represents a decompensated state of brain function resulting from noxious insults overwhelming cerebral reserve. The multifactorial model of delirium describes the interrelationship between baseline risk factors and exposure to precipitating factors leading to delirium. ${ }^{4}$ People with an accumulation of risk factors (older age, dementia, functional impairment, sensory impairment, depression, and alcohol misuse) are more vulnerable to developing delirium from relatively minor noxious insults. ${ }^{4}$ Conversely, people with few or no risk factors require multiple severe insults before their cerebral capacity is overwhelmed. Therefore, older people (with multiple risk factors) and critically ill people (in intensive care units or receiving palliative care) experience the highest rates of delirium. $^{4}$

Despite being commonly encountered by clinicians, the neural mechanisms leading to delirium remain unclear. In the context of the multifactorial model of delirium, it is hypothesized that multiple neural pathways, including neurotransmitter dysregulation, neuroinflammation, impaired functional network connectivity, and abnormal cerebral perfusion and metabolism, are involved. ${ }^{34}$

Due to the clinical overlap and intertwined relationship between delirium and dementia, one way forward is to examine shared pathophysiological substrates (see Figure 1). Most dementia research has focused on Alzheimer's disease (AD), which is the most common type of dementia. The biomarker signature of $\mathrm{AD}$ is brain depositions of amyloid plaques and tau, which are reflected in the cerebrospinal fluid (CSF) as reduced amyloid $\beta$ (A $\beta) 42$ and increased total tau (T-tau) and phosphorylated tau (P-tau). ${ }^{35}$ Few studies have assessed amyloid and tau CSF biomarkers in delirium, and those that have offered inconsistent findings. ${ }^{36}$ Similarly, studies of delirium examining apolipoprotein E (APOE) epsilon 4 genotype, a genetic risk factor strongly associated with late-onset $\mathrm{AD}$, have had conflicting results. ${ }^{37,38}$ One study found that the presence of an APOE4 allele was associated with increased severity of delirium in post-acute care patients with DSD. ${ }^{39}$ Another study measured CSF absolute levels of APOE, which are important for cell repair and neuronal performance. ${ }^{40}$ Patients $>70$ years old with delirium had reduced APOE4 levels compared to patients with $\mathrm{AD}$.

There is substantial evidence to support the idea that neuroinflammation plays a key role in $\mathrm{AD}$ pathogenesis. ${ }^{41}$ Specifically, over time, microglia (the resident macrophage cells in the central nervous system) become "primed", leading to enhanced sensitivity and reactivity to inflammatory precipitants. Chronic exposure leads to sustained microglial cell activation and the release of proinflammatory cytokines, causing structural and functional damage to neurons, which manifests as cognitive decline. ${ }^{41}$ Animal models support the role of neuroinflammation in delirium and demonstrate how noxious inflammatory precipitants, such as sepsis or trauma, lead to the systemic release of cytokines, which, through mechanisms yet to be defined, activate primed microglia, leading to neuronal injury, which, in turn, leads to acute delirium and accelerated dementia. ${ }^{42-44}$ The COVID-19 pandemic has been a tragic natural experiment demonstrating that, in susceptible people, overactivation of the innate immune system, termed cytokine storm ${ }^{45}$ is a determining factor in the development of delirium. This concept is well demonstrated by the high frequency (over 30\%) with which delirium occurs as an onset symptom of COVID-19 in older patients with dementia. ${ }^{46}$

A recent study found that hypometabolism drives cognitive dysfunction in acute systemic inflammation, and restoration of metabolism mitigates cognitive dysfunction in animal models. ${ }^{47}$ Cerebral glucose metabolism is disturbed in both delirium and dementia. ${ }^{48,49}$ An $18^{\mathrm{F}}$ fluorodeoxyglucose positron emission tomography (FDG-PET) scan can be used to distinguish dementia subtypes and predict the conversion of mild cognitive impairment to $\mathrm{AD} .{ }^{50}$ FDG-PET is a sensitive biomarker in AD; hypometabolism predicts progressive cognitive deterioration, even in amyloid-negative populations. ${ }^{51}$ Hypometabolism within the posterior cingulate cortex has been demonstrated during delirium, which improved but did not normalize when delirium resolved. ${ }^{49}$ Cerebral perfusion is closely linked to metabolism, and functional neuroimaging studies demonstrating hypoperfusion during delirium also support abnormal metabolism. ${ }^{52}$ Further studies are required to elucidate the precise mechanisms leading to hypometabolism, for example impaired insulin signaling or compromised glucose transport within the central nervous system. 


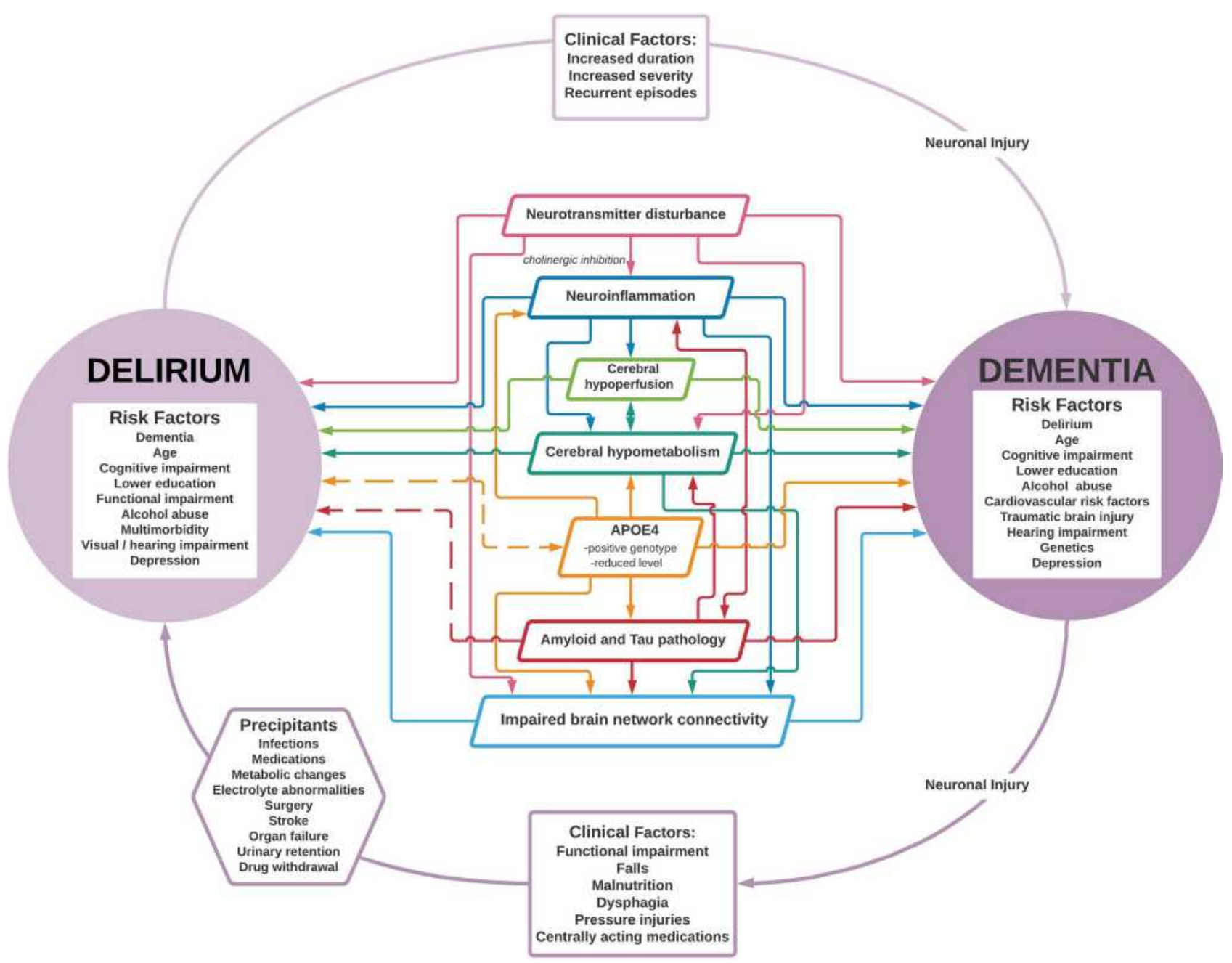

Figure I Pathophysiological links between delirium and dementia. There is significant clinical and pathophysiological overlap between delirium and dementia, particularly in the advanced stages of dementia. Delirium and dementia share many common risk factors and are major risk factors for each other. Exposure to delirium increases the risk of future dementia. Dementia, in turn, increases the risk of developing delirium but also increases exposure to precipitants that cause delirium. There is evidence of neuronal injury during delirium, however, the mechanisms by which this occurs remain unclear. Several hypothesized aberrant pathways may contribute to the development of delirium and dementia. These pathways interact with each other. In delirium, it is likely the impact of each pathway will vary for individual patients depending on baseline vulnerabilities and the type of delirium precipitant. However, a final common pathway may exist accounting for the recognizable syndrome of delirium. APOE4 positive genotyping and amyloid and Tau pathology are strongly associated with Alzheimer's Disease, however, small studies in delirium have demonstrated inconsistent associations. Determining the impact of underlying or preclinical dementia on delirium pathophysiology is challenging but should be considered in all research studies.

Abbreviation: APOE4, apolipoprotein E4.

Recent studies have identified axonal injury during delirium, possibly explaining the sustained cognitive dysfunction that can occur after delirium. Neurofilament light (NfL), which can be measured in both blood and $\mathrm{CSF}$, increases in proportion to the degree of axonal damage. ${ }^{53}$ Elevated NfL levels have been noted in several neurodegenerative diseases, including $\mathrm{AD}$, in which CSF NfL levels predict amyloid load, cerebral atrophy, and cognition. ${ }^{54}$ In an elective surgery cohort, elevated preoperative serum NfL levels predicted delirium, and an elevated NfL level at one month was associated with greater cognitive decline. ${ }^{53}$ Additionally, NfL levels increased in proportion to delirium severity but independently of inflammation. ${ }^{55}$ These findings are consistent with diffusion tensor imaging studies, indicating that post-operative delirium is associated with longitudinal microstructural abnormalities one year after surgery. ${ }^{56}$

\section{Screening and Diagnosis}

The Diagnostic and Statistical Manual of Mental Disorders 5th edition (DSM-5) is the gold standard for diagnosing delirium (with or without dementia). ${ }^{57}$ In the early stages of dementia, diagnosing DSD may be straightforward; however, in advanced dementia, significant clinical overlap 
exists. In reality, diagnosis in this area is often based on elusive foundations because many patients present with delirium but no clinical diagnosis of dementia, whereas even more patients with dementia present with symptoms that may or may not represent delirium. Considering the burden of DSD, few studies have examined screening and diagnosis in this population and existing studies tend to have small sample sizes and are yet to be replicated.

\section{Screening of Delirium Superimposed on Dementia}

There are numerous delirium screening tests and reviewing all in the context of DSD is beyond the scope of this review. ${ }^{58}$ Of note, however, validation studies in DSD populations are sparse and all screening tests have reduced sensitivity and/or specificity when applied to patients with dementia. An in-depth systematic review of DSD tools was conducted in 2012; although 1569 individuals were included, only 50 had DSD. ${ }^{11}$ The confusion assessment method (CAM) was found to be specific (96-100\%) and sensitive $(77 \%)$ in diagnosing DSD. ${ }^{11}$ The CAM is a diagnostic algorithm for delirium detection based on the DSM-III-R criteria, which is widely used in clinical and delirium research settings. ${ }^{59,60}$ Four CAM criteria are judged during patient interviews and cognitive testing: $\mathrm{A}$ ) acute onset and fluctuating course, B) inattention, C) disorganized thinking, and D) level of consciousness. The presence of $\mathrm{A}$ and $\mathrm{B}$ with $\mathrm{C}$ or $\mathrm{D}$ indicates a positive $\mathrm{CAM}$ and is suggestive of delirium. The CAM requires training because it has reduced sensitivity when performed by untrained operators. ${ }^{58}$

Several variations of the CAM now exist for use in specific scenarios. The CAM for the Intensive Care Unit (CAM-ICU) is tailored specifically for patients within the ICU and has demonstrated high sensitivity and specificity in DSD, although sample sizes were small. ${ }^{11,61}$ The 3DCAM is a brief three minute assessment and in patients with dementia was noted to have a sensitivity of $96 \%$ (CI $[82 \%-100 \%])$ and specificity of $86 \%$ (CI [67\% - 96\%]). ${ }^{62}$ The Ultra-Brief two item screener for delirium (UB-2) derived from the 3D-CAM involves two assessment items: "recite the months of year backwards" and "what is the day of the week?". The UB-2 demonstrated high sensitivity $(96 \%$; CI [ $82 \%-100 \%])$ but reduced specificity (43\%; CI [24\% - 63\%]) in DSD populations. ${ }^{63}$

In the advanced stages of dementia specific attention tests may be less reliable, ${ }^{64}$ and there is greater dependence on caregiver collateral history to identify an acute and fluctuating change in cognitive status suggestive of delirium. As such, the Family Confusion Assessment Method (FAM-CAM) was developed to screen for delirium by interviewing family caregivers. ${ }^{65}$ In community dwelling older patients with cognitive impairment $(\mathrm{N}=52)$ the FAM-CAM had a sensitivity of $88 \%$ (CI $[47 \%-99 \%])$ and specificity of $98 \%$ (CI [86\% - 100\%]). ${ }^{65}$

The 4 'A's test (4AT) is commonly used in clinical practice as a delirium screening test. It comprises four items: 1) alertness, 2) the Abbreviated Mental Test-4, 3) an attention test (months of the year backwards), and 4) acute change or fluctuating course. Each item is scored (total $0-12$ ); scores $>3$ are considered positive and indicate possible delirium. ${ }^{66} \mathrm{~A}$ systematic review of the 4AT in older adults ( $>65$ years old) analyzed 17 studies (total $\mathrm{N}=3702) .{ }^{67}$ Pooled sensitivity was $88 \%$, and specificity was $88 \%$. Only three of the included studies reported findings related to known dementia. While the 4AT was found to be sensitive in detecting DSD (86-94\%), specificity was reduced $(65-79 \%) .{ }^{67}$ A recent study suggested that the 4AT has greater overall sensitivity than the CAM and a similarly high level of specificity, although there were insufficient patients with dementia to perform a subgroup analysis for DSD. ${ }^{68}$

Another study aimed to develop a brief screening test to improve health care workers' detection of DSD by focusing on 391 patients $\geq 65$ years old with dementia, with $24 \%$ developing DSD during their hospitalization. ${ }^{69}$ The best single-item screening for DSD was "What day of the week is it?" (sensitivity $84 \%$, CI [0.71 - 0.91] and specificity $41 \%$, CI [0.35 - 0.41]). Extending the screening to three questions ("What day of the week is it?", "List the days of the week backwards", and "Does the patient appear sleepy?") improved the sensitivity but did not impact the specificity. ${ }^{69}$

\section{Diagnosing Delirium in Patients with Dementia}

A critical step in diagnosing DSD is obtaining a collateral history of the patient's baseline cognitive function and behavior to determine whether an acute change has occurred. ${ }^{70}$ In the absence of a collateral history, a patient presenting with cognitive dysfunction or altered level of consciousness should be managed as delirium until proven otherwise. ${ }^{71}$ 


\section{Clinical Assessment}

Inattention and reduced arousal help distinguish delirium from dementia. ${ }^{72,73}$ Attention can be measured by a variety of methods, including stating months of the year backwards, spelling a five-letter word backwards, and digit span backwards. Inattention assessed by asking patients to gesture each time an " $\mathrm{A}$ " is said in S-A-V-E-A-H-A-A-R-T correctly identified DSD 76\% of the time in one study. ${ }^{74}$ Adding the Observation Scale of Level of Arousal in combination with S-A-V-E-A-H-A-A-R-T improved diagnostic accuracy to $93 \%$ in patients with dementia. ${ }^{74}$

The Richmond Agitation and Sedation Scale (RASS) is a validated clinical test that measures arousal, sedation, and level of consciousness in critically ill patients on a scale from -5 (unrousable) to 0 (alert, calm, and attentive) to +4 (combative, violent, and dangerous to staff). ${ }^{75}$ In patients with dementia scoring other than 0 on the RASS and the modified-RASS had a sensitivity of $70.5 \%$ (CI $[65.9 \%-75.1 \%]$ ) and a specificity of $84.8 \%$, (CI $[80.5 \%-89.1 \%])$ for DSD. ${ }^{75}$

Motor dysfunction can also help differentiate DSD from dementia. Tools assessing balance and mobility include the Trunk Control Test and the Hierarchical Assessment of Balance and Mobility. ${ }^{76,77}$

\section{Delirium and Dementia with Lewy Bodies}

The type of underlying dementia may also pose a challenge in diagnosing DSD. Symptoms occurring in the early stages of dementia with Lewy bodies (DLB), such as fluctuations in alertness, visual hallucinations, and rapid eye movement sleep disorders, may mimic delirium. The cognitive profile of mild cognitive impairment with Lewy bodies includes inattention and executive dysfunction, which also occur in delirium. ${ }^{78}$ Furthermore, delirium occurs more frequently prior to the diagnosis of DLB than $\mathrm{AD}(25 \%$ vs $7 \%$ ) and may be a presenting feature of DLB, which is sometimes referred to as delirium onset DLB. ${ }^{78}$ The presence of Parkinsonism, autonomic dysfunction, and neuroleptic sensitivity suggest DLB; however, whenever there is uncertainty, the safest initial action is to assume delirium and investigate and manage accordingly.

\section{Delirium and Behavioral and Psychological Symptoms of Dementia}

The diverse signs and symptoms of BPSD include depression; anxiety; psychosis, including hallucinations and delusions; hyperactivity; aggression apathy; fluctuation, including sundowning; and sleep disturbance. ${ }^{79,80}$ Although there is significant clinical overlap with delirium, BPSD often persists chronically. ${ }^{81}$ Like delirium, BPSD are associated with cognitive decline and progressive dementia. ${ }^{80,82}$ When patients with dementia first present with particularly hyperactive BPSD, a clinical assessment and delirium screen are usually recommended to assess for medically reversible causes. Nonpharmacological management is essentially the same for BPSD and delirium. There is also overlap in pharmacological management, including adequately managing pain and the use of antipsychotics to alleviate distressing psychotic symptoms. Considering the overlap between the conditions, it is possible that they lie on a continuum.

\section{Novel Biomarkers of Delirium Superimposed on Dementia}

While there are no biomarkers for DSD diagnosis, small studies have assessed brain electrical activity and cerebral perfusion.

Electroencephalogram (EEG) is a minimally invasive technique allowing measurement of cerebral electrical activity and thereby assessment of brain synaptic function. Generalized slowing is strongly associated with delirium (odds ratio: 10.3; CI [5.3-20.1]) and the degree of slowing correlates with delirium severity and predicts worse patient outcomes including increased mortality. ${ }^{83}$ With the exception of functional impairment, other risk factors for delirium including age, cognitive impairment, depression and alcohol misuse were not associated with deliriumrelated EEG characteristics in older outpatients awaiting elective surgery. ${ }^{84}$ Therefore, EEG slowing (characterized by increases in delta and theta power and triphasic waves) and reduced functional connectivity can distinguish patients with and without delirium. ${ }^{85}$ EEG also has diagnostic utility particularly to identify mental alterations due to non-convulsive status epilepticus which may present as delirium.

One small study $(\mathrm{N}=35)$ performed electroencephalogram (EEG) tests in older hospitalized patients with dementia; 12 patients had delirium. ${ }^{86}$ Quantitative EEG (eyes open) had moderate sensitivity (67\%) and high specificity (91\%) in identifying DSD. ${ }^{86}$ Overall, EEG may have clinical utility in differentiating delirium from dementia, however, larger studies in this population would be informative.

Another study assessed cerebral perfusion using transcranial Doppler (TCD) to assess blood flow velocity (FV) in the middle cerebral artery. DSD had a lower FV 
than $\mathrm{AD}$ alone, delirium alone, and acute illness. In this population $(\mathrm{N}=44)$, using a mean middle cerebral artery FV cut-off of $32.25 \mathrm{~cm} / \mathrm{s}$, DSD was diagnosed with a specificity of $79 \%$ and a sensitivity of $88 \%{ }^{87}$ Currently, there is insufficient evidence to support use of TCD to help discriminate dementia from DSD.

\section{Investigating Delirium Superimposed on Dementia}

DSD diagnosis is incomplete without investigations to identify the cause(s) of delirium. Older hospitalized patients with delirium usually have multiple precipitating factors contributing to delirium, all of which require identification and management. ${ }^{88}$ A thorough clinical history and examination may identify one or more causes, which are usually confirmed with blood and radiological tests. Common causes of delirium include infections, medications (particularly centrally acting), kidney injury, electrolyte disturbances, metabolic abnormalities, urinary retention, constipation, and stroke. Virtually any medical condition can precipitate delirium in a vulnerable patient. The extent of the investigation is driven by the clinical picture; when the cause of delirium is unclear, further investigations are usually warranted. Over $30 \%$ of older patients presenting to hospitals with delirium have computed tomography (CT) imaging of the brain; however, patients with DSD but no focal neurological signs are likely to have normal scans (predictive value: 0.98 ). ${ }^{89,90}$ Therefore, brain CT should not be routine but should be considered in patients with a history of falls or head injury, focal neurological signs, or anticoagulation and when the cause of delirium is unclear. ${ }^{12}$

There is a need for research into the side effects and cost of overdiagnosis of delirium in nursing home patients with BPSD, including increased bacterial resistance patterns resulting from inappropriate prescription of antibiotics for assumed urinary tract infection.

\section{Identifying Dementia in Patients Presenting with Delirium}

Up to $40 \%$ of hospitalized patients $>70$ years old presenting with delirium have undiagnosed pre-existing cognitive impairment or dementia. ${ }^{91}$ However, since cognitive impairment is also a feature of delirium, its presence is not sufficient for diagnosing dementia, and clinicians should be wary of making an initial diagnosis of dementia in hospitalized patients during acute illness. The neuropsychological profile of delirium is similar with and without comorbid dementia. ${ }^{73}$ Therefore, a collateral history ascertaining performance in individual cognitive domains (attention, executive function, learning and memory, language, perceptual-motor and social cognition) as well as determining the need for assistance with personal and instrumental activities of daily living is informative to help identify probable dementia and facilitate safe discharge planning. Tools, including the Informant Questionnaire of Cognitive Decline in the Elderly and the $\mathrm{AD} 8$, have been validated in delirium and demonstrated high sensitivity and specificity. ${ }^{92}$

\section{Prevention and Management}

There are few studies examining the prevention and management of DSD, however, general delirium management principles should be applied to patients with DSD. Management of delirium focuses on identifying and treating the underlying precipitant(s), creating an ideal environment for brain recovery, preventing complications, managing distress, and monitoring for resolution as well as educating care givers on the possibility of relapse.

Multicomponent interventions aimed at fulfilling patients' essential needs are promoted for the prevention and management of delirium in most clinical guidelines. $^{12,13}$ These interventions are usually interdisciplinary and nonpharmacological; they include regular orientation, cognitive stimulation, optimization of nutrition and hydration, early mobilization, sensory enhancement with the provision of vision and hearing aids, adequate but not excessive pain management, medication review, and optimization of bowel and bladder function. ${ }^{93}$

Almost half of hospital-acquired delirium cases are preventable with nonpharmacological multicomponent intervention (pooled odds ratio: 0.47 ; 95\% CI: $0.38-0.58) .^{94}$ These interventions also reduce falls and are cost effective. ${ }^{94,95}$ Although the evidence that multicomponent interventions alter the trajectory of delirium is not strong, such therapies are fundamentally good practice. $^{93}$

Focusing on studies in DSD populations, preliminary data from a nursing home-based multicomponent intervention included 143 older adults, many with cognitive impairment. $^{96}$ The intervention was feasible, reduced hospital admissions, and resulted in high satisfaction rates for nursing home staff and patients. However, a further clinical trial is required to determine the effect of this intervention on the trajectory of delirium. In a single-blind randomized clinical trial of community-dwelling adults ( $>65$ years old, $\mathrm{N}=283$ ) in post-acute care facilities, 
personalized cognitive stimulation activities did not improve DSD but improved executive function and reduced length of stay. ${ }^{97}$ Since delirium can initiate and accelerate dementia, it is also conceivable that better treatment of delirium would reduce the incidence and progression of dementia; however, evidence for this is yet to be adduced.

A growing body of evidence supports the role of occupational therapy (OT) in the management of delirium. ${ }^{98}$ OT measures include facilitating the use of appropriate bed and chair aids, aiding with safe transfers, assisting with orientation through environmental adaptations, communicating with caregivers and family members, and providing opportunities for cognitive stimulation. ${ }^{99}$ An OT protocol-based intervention providing daily assessment and treatment based on m-RASS scores was found to be feasible for the management of DSD in nursing home residents. ${ }^{99}$ Efficacy studies are now required.

Pharmacological management of delirium with antipsychotics is not routine and should only be used when nonpharmacological strategies have failed. Antipsychotics do not reduce delirium duration, severity, or length of stay and may cause harm. ${ }^{100,101}$ However, expert consensus guides practice and suggests that low-dose antipsychotics may help alleviate distressing symptoms or serve as a chemical restraint when patients are at risk of harming themselves or others.

An essential element of managing patients with DSD is implementing strategies to reduce complications, including pressure area care, fall prevention strategies, and adequate hydration and nutrition. Assisted early mobilization and rehabilitation should be offered to minimize deconditioning.

Communicating with families is essential throughout the hospital stay, and, where feasible, involving family members in caregiving can be beneficial in alleviating the distressing symptoms of delirium. ${ }^{70}$ Early communication and education can improve the caregiver experience and reduce stress. ${ }^{102}$

One of the most challenging aspects of caring for older patients with DSD is the uncertainty of the prognosis. While delirium may resolve within days for some patients, it may persist for months in others. ${ }^{7}$ The causative factors leading to these varied outcomes are not yet known. Thus, for many older patients with dementia, the attainment of a DSD tipping point resulting in increased functional dependence and ultimately institutionalization is severely disabling but clear only in retrospect. Where possible, patients should be given the opportunity to rehabilitate. When this is not feasible, adequate follow-up should be arranged to reassess opportunities for rehabilitation and provide education and support to family members.

\section{Future Directions}

Given the burden of DSD combined with evidence that hospital-acquired delirium is preventable, health care systems should implement evidence-based care standards to overcome long-standing deficiencies in providing holistic care. Delirium is everyone's business, and cognition should be considered a vital sign; just as hypoxia or hypotension compels a clinician to act urgently, so too should the presence of delirium. Adequate training is required across disciplines (medicine, nursing, pharmacy, and allied health) to empower all health care workers to provide person-centered care and share responsibility for delirium screening, prevention, and management.

As emphasized in this paper, the evidence base supporting hypothesized aberrant pathways in delirium is small. This likely reflects the numerous challenges encountered when studying cohorts with delirium, including the transient and fluctuating clinical course, disentangling the contribution of comorbid or preclinical dementia, obstacles in obtaining valid consent, and restrictions in accessing CSF or performing neuroimaging in patients who may be hyperactive and behaviorally challenging. Despite this, considering the far-reaching economic and social costs of delirium, research into its pathophysiology should be prioritised to enable the discovery of biomarkers and potentially targetable neural pathways.

As there is growing evidence of a dose-dependent relationship between delirium and cognitive dysfunction, both clinically ${ }^{3}$ and biochemically (via NfL measurement), ${ }^{53,55}$ research is needed to investigate the mechanisms by which putative neurotoxicity occurs. In the context of the aforementioned challenges, study methodology must be carefully considered. Studies should aim to measure imaging and biomarkers at more than one time point (ideally, at baseline, during- and post-delirium), assess delirium in detail using validated tools, clearly establish baseline and post-delirium cognitive function, and control for confounding variables. ${ }^{36}$ Further examination of shared pathophysiological substrates in delirium and dementia, such as amyloid and tau, as well as neuroinflammation and glucose metabolism may advance our understanding of the interface between the two conditions. 
Studies examining the neuropsychology of delirium and DSD are lacking but have the potential to improve recognition of DSD by clinicians and standardize delirium assessment methods to allow better comparison across studies. ${ }^{103}$ Neuropsychological studies detailing cognitive decline attributable to delirium may help ascertain if there is a specific cognitive profile, such as those found in $\mathrm{AD}$, DLB, and frontotemporal dementia. This would better inform caregivers and assist them with future planning and may help identify neuroanatomical substrates that are preferentially affected during delirium.

\section{Conclusion}

DSD is commonly encountered by clinicians and associated with multiple adverse sequelae, including accelerated dementia. Inattention, altered arousal, and motor dysfunction may help differentiate delirium from dementia; however, where there is diagnostic uncertainty, it is safest to assume delirium until proven otherwise. When available, family and informal caregivers often provide critical information to help detect DSD and also assist in management. The neural mechanisms causing delirium and subsequent cognitive decline are not yet known and must become a priority in agerelated research. Given the clinical overlap between delirium and dementia, examining shared pathophysiological substrates may be helpful in developing diagnostic tests, and identifying potentially targetable therapeutic pathways. The development of therapeutics for the prevention and treatment of delirium has the potential to not only improve outcomes for patients but also reduce the global dementia burden.

\section{Disclosure}

The authors report no conflicts of interest in this work.

\section{References}

1. American Psychiatric Association. Diagnostic and Statistical Manual of Mental Disorders: DSM-5. Arlington, VA: American Psychiatric Association; 2013.

2. Vanz-Brian Pereira J, Aung Thein MZ, Nitchingham A, Caplan GA. Delirium in older adults is associated with development of new dementia: a systematic review and meta-analysis. Int $J$ Geriatr Psychiatry. 2021. doi:10.1002/gps.5508

3. Richardson SJ, Davis DHJ, Stephan BCM, et al. Recurrent delirium over 12 months predicts dementia: results of the Delirium and Cognitive Impact in Dementia (DECIDE) study. Age Ageing. 2020. doi:10.1093/ageing/afaa244

4. Inouye SK, Westendorp RG, Saczynski JS. Delirium in elderly people. Lancet. 2014;383(9920):911-922. doi:10.1016/S0140-6736(13)606881

5. Robertsson B, Blennow K, Gottfries C, Wallin A. Delirium in dementia. Int J Geriatr Psychiatry. 1998;13(1):49-56. doi:10.1002/ (SICI)1099-1166(199801)13:1<49::AID-GPS733>3.0.CO;2-4
6. Cole MG, Ciampi A, Belzile E, Zhong L. Persistent delirium in older hospital patients: a systematic review of frequency and prognosis. Age Ageing. 2008;38(1):19-26. doi:10.1093/ageing/afn253

7. Cole MG, Bailey R, Bonnycastle M, et al. Partial and no recovery from delirium in older hospitalized adults: frequency and baseline risk factors. $J$ Am Geriatr Soc. 2015;63(11):2340-2348. doi:10.1111/jgs.13791

8. Kakuma R, Du Fort GG, Arsenault L, et al. Delirium in Older Emergency Department Patients Discharged Home: effect on Survival. J Am Geriatr Soc. 2003;51(4):443-450. doi:10.1046/ j.1532-5415.2003.51151.x

9. Collins N, Blanchard MR, Tookman A, Sampson EL. Detection of delirium in the acute hospital. Age Ageing. 2010;39 (1):131-135. doi:10.1093/ageing/afp201

10. Caplan GA. Delirium Superimposed Upon Dementia. J Am Med Dir Assoc. 2019;20(11):1382-1383. doi:10.1016/j. jamda.2019.09.019

11. Morandi A, McCurley J, Vasilevskis EE, et al. Tools to Detect Delirium Superimposed on Dementia: a Systematic Review. J Am Geriatr Soc. 2012;60(11):2005-2013. doi:10.1111/j.15325415.2012.04199.x

12. Scottish Intercollegiate Guidelines Network (SIGN). Risk Reduction and Management of Delirium. Edinburgh: Scottish Intercollegiate Guidelines Network (SIGN); 2019.

13. Australian Commission on Safety and Quality in Health Care. Delirium Clinical Care Standard. Sydney: Australian Commission on Safety and Quality in Health Care; 2016.

14. Gibb K, Seeley A, Quinn T, et al. The consistent burden in published estimates of delirium occurrence in medical inpatients over four decades: a systematic review and meta-analysis study. Age Ageing. 2020;49(3):352-360. doi:10.1093/ageing/afaa040

15. Mukadam N, Sampson EL. A systematic review of the prevalence, associations and outcomes of dementia in older general hospital inpatients. Int Psychogeriatrics. 2011;23(3):344. doi:10.1017/S1041610210001717

16. Fick DM, Agostini JV, Inouye SK. Delirium Superimposed on Dementia: a Systematic Review. J Am Geriatr Soc. 2002;50 (10):1723-1732. doi:10.1046/j.1532-5415.2002.50468.x

17. Avelino-Silva TJ, Campora F, Curiati JAE, Jacob-Filho W. Association between delirium superimposed on dementia and mortality in hospitalized older adults: a prospective cohort study. PLoS Med. 2017;14(3):e1002264. doi:10.1371/journal. pmed.1002264

18. Morandi A, Di Santo SG, Zambon A, et al. Delirium, Dementia, and In-Hospital Mortality: the Results From the Italian Delirium Day 2016, A National Multicenter Study. J Gerontology. 2019;74 (6):910-916. doi:10.1093/gerona/gly154

19. Sampson EL, Blanchard MR, Jones L, Tookman A, King M. Dementia in the acute hospital: prospective cohort study of prevalence and mortality. Br J Psychiatry. 2009;195(1):61-66. doi:10.1192/bjp.bp.108.055335

20. McCusker J, Cole M, Abrahamowicz M, Primeau F, Belzile E. Delirium predicts 12-month mortality. Arch Intern Med. 2002;162 (4):457-463. doi:10.1001/archinte.162.4.457

21. Witlox J, Eurelings LS, de Jonghe JF, Kalisvaart KJ, Eikelenboom P, van Gool WA. Delirium in elderly patients and the risk of postdischarge mortality, institutionalization, and dementia: a meta-analysis. JAMA. 2010;304(4):443-451. doi:10.1001/jama.2010.1013

22. Siddiqi N, House AO, Holmes JD. Occurrence and outcome of delirium in medical in-patients: a systematic literature review. Age Ageing. 2006;35(4):350-364. doi:10.1093/ageing/afl005

23. Aung Thein MZ, Pereira JV, Nitchingham A, Caplan GA. A call to action for delirium research: meta-analysis and regression of delirium associated mortality. BMC Geriatr. 2020;20(1). doi:10.1186/s12877-020-01723-4 
24. Fick DM, Steis MR, Waller JL, Inouye SK. Delirium superimposed on dementia is associated with prolonged length of stay and poor outcomes in hospitalized older adults. $J$ Hospital Med. 2013;8(9):500-505. doi:10.1002/jhm.2077

25. Bellelli G, Frisoni GB, Turco R, Lucchi E, Magnifico F, Trabucchi M. Delirium Superimposed on Dementia Predicts 12-Month Survival in Elderly Patients Discharged From a Postacute Rehabilitation Facility. J Gerontology. 2007;62 (11):1306-1309. doi:10.1093/gerona/62.11.1306

26. Reynish EL, Hapca SM, De Souza N, Cvoro V, Donnan PT, Guthrie B. Epidemiology and outcomes of people with dementia, delirium, and unspecified cognitive impairment in the general hospital: prospective cohort study of 10,014 admissions. BMC Med. 2017;15(1). doi:10.1186/s12916-017-0899-0

27. Gual N, Morandi A, Pérez LM, et al. Risk Factors and Outcomes of Delirium in Older Patients Admitted to Postacute Care with and without Dementia. Dement Geriatr Cogn Disord. 2018;45 (1-2):121-129. doi:10.1159/000485794

28. Gross AL, Jones RN, Habtemariam DA, et al. Delirium and Long-term Cognitive Trajectory Among Persons With Dementia. Arch Intern Med. 2012;172(17):1324. doi:10.1001/ archinternmed.2012.3203

29. Pezzullo L, Streatfeild J, Hickson J, Teodorczuk A, Agar MR, Caplan GA. Economic impact of delirium in Australia: a cost of illness study. BMJ Open. 2019;9(9):e027514. doi:10.1136/bmjopen-2018-027514

30. Fick DM, Kolanowski AM, Waller JL, Inouye SK. Delirium superimposed on dementia in a community-dwelling managed care population: a 3-year retrospective study of occurrence, costs, and utilization. J Gerontol a Biol Sci Med Sci. 2005;60 (6):748-753. doi:10.1093/gerona/60.6.748

31. Grossi E, Lucchi E, Gentile S, Trabucchi M, Bellelli G, Morandi A. Preliminary investigation of predictors of distress in informal caregivers of patients with delirium superimposed on dementia. Aging Clin Exp Res. 2020;32(2):339-344. doi:10.1007/s40520-019-01194-7

32. Morandi A, Lucchi E, Turco R, et al. Delirium superimposed on dementia: a quantitative and qualitative evaluation of patient experience. J Psychosom Res. 2015;79(4):281-287. doi:10.1016/ j.jpsychores.2015.07.010

33. Morandi A, Lucchi E, Turco R, et al. Delirium superimposed on dementia: a quantitative and qualitative evaluation of informal caregivers and health care staff experience. J Psychosom Res. 2015;79(4):272-280. doi:10.1016/j.jpsychores.2015.06.012

34. Maldonado JR. Delirium pathophysiology: an updated hypothesis of the etiology of acute brain failure. Int $J$ Geriatr Psychiatry. 2018;33(11):1428-1457. doi:10.1002/gps.4823

35. Olsson B, Lautner R, Andreasson U, et al. CSF and blood biomarkers for the diagnosis of Alzheimer's disease: a systematic review and meta-analysis. Lancet Neurology. 2016;15 (7):673-684. doi:10.1016/S1474-4422(16)00070-3

36. Fong TG, Vasunilashorn SM, Libermann T, Marcantonio ER, Inouye SK. Delirium and A lzheimer disease: a proposed model for shared pathophysiology. Int J Geriatr Psychiatry. 2019;34 (6):781-789. doi:10.1002/gps.5088

37. van Munster BC, Korevaar JC, Zwinderman AH, Leeflang MM, de Rooij SE. The association between delirium and the apolipoprotein E epsilon 4 allele: new study results and a meta-analysis. Am J Geriatric Psychiatry. 2009;17(10):856-862. doi:10.1097/ JGP.0b013e3181ab8c84

38. Adamis D, Meagher D, Williams J, Mulligan O, McCarthy G. A systematic review and meta-analysis of the association between the apolipoprotein E genotype and delirium. Psychiatr Genet. 2016;26(2):53-59. doi:10.1097/YPG.0000000000000122

39. Massimo L, Munoz E, Hill N, et al. Genetic and environmental factors associated with delirium severity in older adults with dementia. Int J Geriatr Psychiatry. 2017;32(5):574-581. doi:10.1002/gps.4496
40. Caplan GA, Tai J, Mohd Hanizan F, McVeigh C, Hill M, Poljak A. Cerebrospinal Fluid Apolipoprotein E Levels in Delirium. Dement Geriatr Cogn Dis Extra. 2017;7(2):240-248. doi: $10.1159 / 000477847$

41. Heneka MT, Carson MJ, Khoury JE, et al. Neuroinflammation in Alzheimer's disease. Lancet Neurology. 2015;14(4):388-405.

42. Hennessy E, Gormley S, Lopez-Rodriguez AB, Murray C, Murray C, Cunningham C. Systemic TNF- $\alpha$ produces acute cognitive dysfunction and exaggerated sickness behavior when superimposed upon progressive neurodegeneration. Brain Behav Immun. 2017;59:233-244. doi:10.1016/j.bbi.2016.09.011

43. Wilson JE, Mart MF, Cunningham C, et al. Delirium. Nat Rev Dis Primers. 2020;6(1):54.

44. Wang P, Velagapudi R, Kong C, et al. Neurovascular and immune mechanisms that regulate postoperative delirium superimposed on dementia. AlzheimerDementia. 2020;16(5):734-749. doi:10.1002/alz.12064

45. Nidadavolu LS, Walston JD. Underlying Vulnerabilities to the Cytokine Storm and Adverse COVID-19 Outcomes in the Aging Immune System. J Gerontology. 2021;76(3):e13-e18. doi:10.1093/gerona/glaa209

46. Poloni TE, Carlos AF, Cairati M, et al. Prevalence and prognostic value of Delirium as the initial presentation of COVID-19 in the elderly with dementia: an Italian retrospective study. EClinicalMedicine. 2020;26:100490. doi:10.1016/j.eclinm.2020.100490

47. Kealy J, Murray C, Griffin EW, et al. Acute Inflammation Alters Brain Energy Metabolism in Mice and Humans: role in Suppressed Spontaneous Activity, Impaired Cognition, and Delirium. J Neurosci. 2020;40(29):5681-5696. doi:10.1523/ JNEUROSCI.2876-19.2020

48. Kellar D, Craft S. Brain insulin resistance in Alzheimer's disease and related disorders: mechanisms and therapeutic approaches. Lancet Neurology. 2020;19(9):758-766. doi:10.1016/S14744422(20)30231-3

49. Haggstrom LR, Nelson JA, Wegner EA, Caplan GA. 2-18F-fluoro -2-deoxyglucose positron emission tomography in delirium. $J$ Cerebral Blood Flow Metab. 2017;37(11):3556-3567. doi:10.1177/0271678X17701764

50. Mosconi L, Tsui WH, Herholz K, et al. Multicenter Standardized 18F-FDG PET Diagnosis of Mild Cognitive Impairment, Alzheimer's Disease, and Other Dementias. $J$ Nucl Med. 2008;49(3):390-398. doi:10.2967/jnumed.107.045385

51. Chételat G, Arbizu J, Barthel H, et al. Amyloid-PET and 18FFDG-PET in the diagnostic investigation of Alzheimer's disease and other dementias. Lancet Neurology. 2020;19(11):951-962. doi:10.1016/S1474-4422(20)30314-8

52. Nitchingham A, Kumar V, Shenkin S, Ferguson KJ, Caplan GA. A systematic review of neuroimaging in delirium: predictors, correlates and consequences. Int J Geriatr Psychiatry. 2018;33 (11):1458-1478. doi:10.1002/gps.4724

53. Fong TG, Vasunilashorn SM, Ngo L, et al. Association of Plasma Neurofilament Light with Postoperative Delirium. Ann Neurol. 2020;88(5):984-994. doi:10.1002/ana.25889

54. Dhiman K, Gupta VB, Villemagne VL, et al. Cerebrospinal fluid neurofilament light concentration predicts brain atrophy and cognition in Alzheimer's disease. AlzheimerDementia. 2020;12(1).

55. Casey CP, Lindroth H, Mohanty R, et al. Postoperative delirium is associated with increased plasma neurofilament light. Brain. 2020;143(1):47-54. doi:10.1093/brain/awz354

56. Cavallari M, Dai W, Guttmann CRG, et al. Longitudinal diffusion changes following postoperative delirium in older people without dementia. Neurology. 2017;89(10):1020-1027. doi:10.1212/ WNL.0000000000004329

57. Richardson S, Teodorczuk A, Bellelli G, et al. Delirium superimposed on dementia: a survey of delirium specialists shows a lack of consensus in clinical practice and research studies. Int Psychogeriatrics. 2016;28 (5):853-861. doi:10.1017/S1041610215002288 
58. De J, Wand APF. Delirium Screening: a Systematic Review of Delirium Screening Tools in Hospitalized Patients. The Gerontologist. 2015;55(6):1079-1099. doi:10.1093/geront/gnv100

59. Inouye SK. The Short Confusion Assessment Method (Short CAM): Training Manual and Coding Guide. Boston: Hospital Elder Life Program; 2014.

60. Inouye $\mathrm{SK}$, van Dyck $\mathrm{CH}$, Alessi CA, Balkin S, Siegal AP, Horwitz RI. Clarifying confusion: the confusion assessment method. A new method for detection of delirium. Ann Intern Med. 1990;113(12):941-948. doi:10.7326/0003-4819-113-12-941

61. Ely EW, Inouye SK, Bernard GR, et al. Delirium in mechanically ventilated patients: validity and reliability of the confusion assessment method for the intensive care unit (CAM-ICU). JAMA. 2001;286(21):2703-2710. doi:10.1001/jama.286.21.2703

62. Marcantonio ER, Ngo LH, O'Connor M, et al. 3D-CAM: derivation and Validation of a 3-Minute Diagnostic Interview for CAM-Defined Delirium. Ann Intern Med. 2014;161(8):554. doi:10.7326/M14-0865

63. Fick DM, Inouye SK, Guess J, et al. Preliminary development of an ultrabrief two-item bedside test for delirium. $J$ Hospital Med. 2015;10(10):645-650. doi:10.1002/jhm.2418

64. Voyer P, Champoux N, Desrosiers J, et al. Assessment of inattention in the context of delirium screening: one size does not fit all! Int Psychogeriatrics. 2016;28(8):1293-1301. doi:10.1017/ S1041610216000533

65. Steis MR, Evans L, Hirschman KB, et al. Screening for Delirium Using Family Caregivers: convergent Validity of the Family Confusion Assessment Method and Interviewer-Rated Confusion Assessment Method. J Am Geriatr Soc. 2012;60(11):2121-2126. doi:10.1111/j.1532-5415.2012.04200.x

66. Bellelli G, Morandi A, Davis DHJ, et al. Validation of the 4AT, a new instrument for rapid delirium screening: a study in 234 hospitalised older people. Age Ageing. 2014;43(4):496-502. doi:10.1093/ageing/afu021

67. Tieges Z, Maclullich AMJ, Anand A, et al. Diagnostic accuracy of the 4AT for delirium detection in older adults: systematic review and meta-analysis. Age Ageing. 2020. doi:10.1093/ageing/afaa224

68. Shenkin SD, Fox C, Godfrey M, et al. Delirium detection in older acute medical inpatients: a multicentre prospective comparative diagnostic test accuracy study of the 4AT and the confusion assessment method. BMC Med. 2019;17(1). doi:10.1186/s12916019-1367-9

69. Steensma E, Zhou W, Ngo L, et al. Ultra-brief Screeners for Detecting Delirium Superimposed on Dementia. J Am Med Dir Assoc. 2019;20 (11):1391-1396.e1391. doi:10.1016/j.jamda.2019.05.011

70. Shrestha P, Fick DM. Family caregiver's experience of caring for an older adult with delirium: a systematic review. Int $J$ Older People Nurs. 2020;15(4). doi:10.1111/opn.12321

71. Morandi A, Davis D, Bellelli G, et al. The Diagnosis of Delirium Superimposed on Dementia: an Emerging Challenge. J Am Med Dir Assoc. 2017;18(1):12-18. doi:10.1016/j.jamda.2016.07.014

72. Leonard M, O'Connell H, Williams O, et al. Attention, vigilance and visuospatial function in hospitalized elderly medical patients: relationship to neurocognitive diagnosis. J Psychosom Res. 2016;90:84-90. doi:10.1016/j.jpsychores.2016.09.011

73. Leonard M, McInerney S, McFarland J, et al. Comparison of cognitive and neuropsychiatric profiles in hospitalised elderly medical patients with delirium, dementia and comorbid delirium-dementia. BMJ Open. 2016;6(3):e009212. doi:10.1136/ bmjopen-2015-009212

74. Richardson SJ, Davis DHJ, Bellelli G, et al. Detecting delirium superimposed on dementia: diagnostic accuracy of a simple combined arousal and attention testing procedure. Int Psychogeriatrics. 2017;29(10):1585-1593. doi:10.1017/S1041610217000916
75. Morandi A, Han JH, Meagher D, et al. Detecting Delirium Superimposed on Dementia: evaluation of the Diagnostic Performance of the Richmond Agitation and Sedation Scale. $J$ Am Med Dir Assoc. 2016;17(9):828-833. doi:10.1016/j. jamda.2016.05.010

76. Gual N, Richardson SJ, Davis DHJ, et al. Impairments in balance and mobility identify delirium in patients with comorbid dementia. Int Psychogeriatrics. 2018;31(5):749-753. doi:10.1017/S1041610218001345

77. Bellelli G, Speciale S, Morghen S, Torpilliesi T, Turco R, Trabucchi M. Are Fluctuations in Motor Performance a Diagnostic Sign of Delirium? J Am Med Dir Assoc. 2011;12 (8):578-583. doi:10.1016/j.jamda.2010.04.010

78. McKeith IG, Ferman TJ, Thomas AJ, et al. Research criteria for the diagnosis of prodromal dementia with Lewy bodies. Neurology. 2020;94(17):743-755. doi:10.1212/ WNL.0000000000009323

79. van der Linde RM, Dening T, Matthews FE, Brayne C. Grouping of behavioural and psychological symptoms of dementia. Int J Geriatr Psychiatry. 2014;29(6):562-568. doi:10.1002/gps.4037

80. Tible OP, Riese F, Savaskan E, Von Gunten A. Best practice in the management of behavioural and psychological symptoms of dementia. Ther Adv Neurol Disord. 2017;10(8):297-309. doi:10.1177/1756285617712979

81. Van Der Linde RM, Dening T, Stephan BCM, Prina AM, Evans E, Brayne C. Longitudinal course of behavioural and psychological symptoms of dementia: systematic review. $\mathrm{Br} J$ Psychiatry. 2016;209(5):366-377. doi:10.1192/bjp.bp.114.148403

82. Witlox J, Eurelings L, de Jonghe J, Kalisvaart K, Eikelenboom P, van Gool W. Delirium in Elderly Patients and the Risk of Postdischarge Mortality, Institutionalization, and Dementia A Meta-analysis. JAMA J Am Med Assoc. 2010;304(4):443-451.

83. Kimchi EY, Neelagiri A, Whitt W, et al. Clinical EEG slowing correlates with delirium severity and predicts poor clinical outcomes. Neurology. 2019;93(13):e1260-e1271. doi:10.1212/ WNL.0000000000008164

84. Van Montfort SJT, Van Dellen E, Wattel LL, et al. Predisposition for delirium and EEG characteristics. Clin Neurophysiology. 2020;131(5):1051-1058. doi:10.1016/j.clinph.2020.01.023

85. Boord MS, Moezzi B, Davis D, et al. Investigating how electroencephalogram measures associate with delirium: a systematic review. Clin Neurophysiology. 2021;132(1):246-257. doi:10.1016/j.clinph.2020.09.009

86. Thomas C, Hestermann U, Walther S, et al. Prolonged activation EEG differentiates dementia with and without delirium in frail elderly patients. J Neurol Neurosurg Psychiatry. 2008;79 (2):119-125. doi:10.1136/jnnp.2006.111732

87. Caplan GA, Lan Z, Newton L, Kvelde T, McVeigh C, Hill MA. Transcranial Doppler to measure cerebral blood flow in delirium superimposed on dementia. A cohort study. J Am Med Dir Assoc. 2014;15(5):355-360. doi:10.1016/j.jamda.2013.12.079

88. Laurila JV, Laakkonen M-L, Laurila JV, Timo SE, Reijo TS. Predisposing and precipitating factors for delirium in a frail geriatric population. J Psychosom Res. 2008;65(3):249-254. doi:10.1016/j.jpsychores.2008.05.026

89. Hijazi Z, Lange P, Watson R, Maier AB. The use of cerebral imaging for investigating delirium aetiology. Eur J Intern Med. 2018;52:35-39. doi:10.1016/j.ejim.2018.01.024

90. Hufschmidt A, Shabarin V. Diagnostic yield of cerebral imaging in patients with acute confusion. Acta Neurol Scand. 2008;118 (4):245-250. doi:10.1111/j.1600-0404.2008.01006.x

91. Jackson TA, Maclullich AMJ, Gladman JRF, Lord JM, Sheehan B. Undiagnosed long-term cognitive impairment in acutely hospitalised older medical patients with delirium: a prospective cohort study. Age Ageing. 2016;45(4):493-499. doi:10.1093/ageing/afw064 
92. Jackson TA, Maclullich AMJ, Gladman JRF, Lord JM, Sheehan B. Diagnostic test accuracy of informant-based tools to diagnose dementia in older hospital patients with delirium: a prospective cohort study. Age Ageing. 2016;45(4):505-511. doi:10.1093/ageing/afw065

93. Oh ES, Fong TG, Hshieh TT, Inouye SK. Delirium in Older Persons: advances in Diagnosis and Treatment. JAMA. 2017;318 (12):1161-1174. doi:10.1001/jama.2017.12067

94. Hshieh TT, Yue J, Oh E, et al. Effectiveness of Multicomponent Nonpharmacological Delirium Interventions. JAMA Intern Med. 2015;175(4):512. doi:10.1001/jamainternmed.2014.7779

95. Caplan GA, Harper EL. Recruitment of volunteers to improve vitality in the elderly: the REVIVE* study. Intern Med J. 2007;37 (2):95-100. doi:10.1111/j.1445-5994.2007.01265.x

96. Boockvar KS, Teresi JA, Inouye SK. Preliminary Data: an Adapted Hospital Elder Life Program to Prevent Delirium and Reduce Complications of Acute Illness in Long-Term Care Delivered by Certified Nursing Assistants. J Am Geriatr Soc. 2016;64(5):1108-1113. doi:10.1111/jgs.14091

97. Kolanowski A, Fick D, Litaker M, et al. Effect of Cognitively Stimulating Activities on Symptom Management of Delirium Superimposed on Dementia: a Randomized Controlled Trial. J Am Geriatr Soc. 2016;64(12):2424-2432. doi:10.1111/jgs.14511

98. Pozzi C, Lucchi E, Lanzoni A, et al. Preliminary Evidence of a Positive Effect of Occupational Therapy in Patients With Delirium Superimposed on Dementia. J Am Med Dir Assoc. 2017;18(12):1091-1092. doi:10.1016/j.jamda.2017.09.005
99. Pozzi C, Lanzoni A, Lucchi E, et al. Activity-based occupational therapy intervention for delirium superimposed on dementia in nursing home setting: a feasibility study. Aging Clin Exp Res. 2020;32(5):827-833. doi:10.1007/s40520-019-01422-0

100. Neufeld KJ, Yue J, Robinson TN, Inouye SK, Needham DM. Antipsychotic Medication for Prevention and Treatment of Delirium in Hospitalized Adults: a Systematic Review and Meta-Analysis. $J$ Am Geriatr Soc. 2016;64(4):705-714. doi:10.1111/jgs.14076

101. Agar MR, Lawlor PG, Quinn S, et al. Efficacy of oral risperidone, haloperidol, or placebo for symptoms of delirium among patients in palliative care: a randomized clinical trial. JAMA Intern Med. 2017;177(1):34-42. doi:10.1001/jamainternmed.2016.7491

102. Partridge JS, Martin FC, Harari D, Dhesi JK. The delirium experience: what is the effect on patients, relatives and staff and what can be done to modify this? Int $J$ Geriatr Psychiatry. 2013;28(8):804-812. doi:10.1002/gps.3900

103. Tieges Z, Evans JJ, Neufeld KJ, MacLullich AMJ. The neuropsychology of delirium: advancing the science of delirium assessment. Int $J$ Geriatr Psychiatry. 2018;33(11):1501-1511. doi:10.1002/gps.4711

\section{Publish your work in this journal}

Neuropsychiatric Disease and Treatment is an international, peerreviewed journal of clinical therapeutics and pharmacology focusing on concise rapid reporting of clinical or pre-clinical studies on a range of neuropsychiatric and neurological disorders. This journal is indexed on PubMed Central, the 'PsycINFO' database and CAS, and is the official journal of The International Neuropsychiatric Association (INA). The manuscript management system is completely online and includes a very quick and fair peer-review system, which is all easy to use. Visit http://www.dovepress.com/testimonials.php to read real quotes from published authors. 